\title{
sciendo
}

\section{The Effects of Altering the Concentric/Eccentric Phase Times on EMG Response, Lactate Accumulation and Work Completed when Training to Failure}

\author{
by \\ Gustavo Ferreira Pedrosa, ${ }^{1,2}$, Sandra Carvalho Machado ${ }^{1}$, \\ Rodrigo César Ribeiro Diniz', Lucas Túlio de Lacerda1,3,4, \\ Hugo Cesar Martins-Costa ${ }^{1,4}$, André Gustavo Pereira de Andrade ${ }^{5}$, \\ Michael Bemben ${ }^{6}$, Mauro Heleno Chagas ${ }^{1}$, Fernando Vitor Lima ${ }^{1}$
}

This study compared the electromyographic response, the blood lactate concentration (BLC), and the maximum number of repetitions (MNR) between protocols of different concentricleccentric duration taken to muscle failure. This comparison may help to understand how different concentricleccentric duration may influence performance and the central and metabolic responses in trained men. Seventeen strength-trained men performed two protocols in a counterbalanced design. Three sets of the Smith bench press exercise were performed to failure at $60 \%$ of the onerepetition maximum (1RM) using each protocol (4-s concentric/2-s eccentric [4 s: $2 \mathrm{~s}$; and 2-s concentric/4-s eccentric [2 s: $4 \mathrm{~s}]$ ). The normalized root mean square (EMGRMS) and the mean frequency (EMGMF) of the electromyographic signals for the pectoralis major and the triceps brachii were compared in the first, middle, and last repetitions. The BLC was assessed at rest, during and after the test sessions. To compare the EMG and BLC, a 3-way ANOVA with repeated measures with a post hoc Tukey's test was used. To compare the MNR performed across the sets, an ANOVA-type rank test with the Dunn's post hoc test was used. The ANOVA indicated a greater EMGRMS for Protocol $4 \mathrm{~s}: 2 \mathrm{~s}$ in the pectoralis major and a lower EMGMF for Protocol $4 \mathrm{~s}: 2 \mathrm{~s}$ in the triceps brachii at the middle and last repetitions. Both protocols increased the EMGRMS and decreased the EMGMF across repetitions. Despite the results show different levels of activation and neuromuscular fatigue between protocols, the BLC and the MNR were similar.

Key words: physiology, strength, electromyography, bench press, central fatigue.

\section{Introduction}

Strength training programs are generally designed to induce functional and morphologic adaptations, which are fundamental to many sports (Batalha et al., 2018; Lago-Fuentes et al., 2018; Maszczyk et al., 2020). Different strength

\footnotetext{
1 - Weight Training Laboratory, School of Physical Education, Physiotherapy and Occupational Therapy of the Federal University of Minas Gerais, Belo Horizonte, Brazil.

2 - Centro de Instrução e Adaptação da Aeronáutica, Lagoa Santa, Brazil.

3 - Department of Physical Education and Sports, Technological Education Federal Center of Minas Gerais, Belo Horizonte, Minas Gerais, Brazil.

4 - Laboratory of Movement Analysis and Human Performance, Department of Physical Education, Pontifical Catholic University of Minas Gerais, Belo Horizonte, Brazil.

5 - Biomechanical Laboratory of Physical Education, Physiotherapy and Occupational Therapy of the Federal University of Minas Gerais, Belo Horizonte, Brazil.

6 - Department of Health and Exercise Science. University of Oklahoma, Norman, United States.
} 
training configurations may cause different neuromuscular responses, and consequently, impact the muscle's capacity to produce force (Jenkins et al., 2015). Thus, physical trainers and coaches need to match the athlete's objective with a proper strength training configuration, with the aim of improving the athlete's performance. The strength training configuration is based on multiple variable combinations (e.g., volume, intensity, and frequency) (Ratamess et al., 2009). Therefore, understanding how the physiological effects manifest when each variable is manipulated may contribute to the sports professional to prescribe training routines more suitable for their respective athletes.

The repetition duration is a variable prescribed in strength training programs (Usui et al., 2016). However, little attention has been given to the configuration of the time spent in each phase (concentric or eccentric) during a given contraction, despite evidence that this can influence the strength training adaptations (Usui et al., 2016). In a study by Lacerda et al. (2017), the electromyographic (EMG) amplitude and the EMG mean frequencies (EMGMF) were compared between two protocols configured with different muscle action duration, but with the same total repetition duration (6s). In the first protocol, each repetition was executed with a $4 \mathrm{~s}$ concentric phase and a 2 s eccentric phase (Protocol 4 s: 2 s), whereas in the second protocol, each repetition was performed with a $2 \mathrm{~s}$ concentric phase and a 4 $\mathrm{s}$ eccentric phase (Protocol $2 \mathrm{s:} 4 \mathrm{~s}$ ). Both protocols utilized 3 sets with 6 repetitions at $60 \%$ of the onerepetition maximum (1RM) with a 3-min rest interval between sets during the Smith machine bench press exercise. In that study, the protocol with the longer concentric duration resulted in greater EMG amplitudes and lower EMGMF responses than the protocol with the longer eccentric duration. Thus, the authors concluded that performing longer concentric contractions could be a more appropriate strategy for increasing muscle activation and neuromuscular fatigue, which was in line with other researchers (Fang et al., 2001). The increase in EMG amplitude is linked, at least in part, to an increase in higher threshold type II motor recruitment (Hunter et al., 2004), which is able to achieve greater levels of force production. Thus, more time spent during the concentric action than in the eccentric action may be an effective strategy to stimulate these fibres. Given the expectation that the activated muscle fibres are those that adapt to training (Wakahara et al., 2013), protocols that show a higher EMG amplitude could present greater potential to promote increases in strength and muscle hypertrophy (Watanabe et al., 2014).

However, the two protocols cited above were not designed to achieve muscular failure, which may have maximized the concentric phase and led to a distinct neuromuscular response. In a study by Goto et al. (2009), in which the protocols were completed to failure, the protocol with the longer concentric duration phase (Protocol $5 \mathrm{~s}: 1 \mathrm{~s}$ ) resulted in a lower maximum number of repetitions (MNR) when compared to a protocol with longer eccentric duration (Protocol $1 \mathrm{~s}: 5 \mathrm{~s}$ ). These results indicated that even though the total duration time of the contractions was equivalent, less work was done when there were longer concentric contraction times. When an exercise is performed until muscle failure, neuromuscular strategies that involve the additional recruitment of higher threshold type II motor units may occur in an attempt to preserve the desired force (Hunter et al., 2004), which results in a greater decrease of EMGMF (Brody et al., 1991). Following this line of reasoning, it could be hypothesized that if the Lacerda et al.'s (2017) protocols were performed until muscle failure, perhaps Protocol $2 \mathrm{~s}: 4 \mathrm{~s}$ might have produced a greater number of repetitions and the differences in the EMG amplitude and EMGMF response may have been attenuated or even similar. However, this has not been analyzed yet.

Furthermore, greater EMG amplitude and reduced EMGMF response seem to be associated with greater blood lactate concentrations (BLCs) (Lacerda et al., 2016). In the study by Goto et al. (2009), Protocol 5 s: 1 s resulted in a greater BLC response than Protocol 1 s: 5 s. However, the EMG response was not investigated. In addition, the protocols used by Goto et al. (2009) had a large 4-s difference between the concentric and eccentric muscle action duration. Perhaps protocols configured with smaller differences between the muscle action duration, but with the same repetition duration as the two protocols of Lacerda et al. (2017) if taken to muscle failure, might produce a similar BLC response.

It is not clear if protocols with small 
differences in the time spent in each muscle action (2 s) could provide differences in the EMG, BLC, and the MNR performed. This information may also provide a basis for a prescription of the training load, which may promote a varied stimulus to the organism from an alteration still requiring further analysis. Therefore, this study aimed to compare the EMG amplitude, EMGMF, BLC response, and the MNR between and within protocols differentiated by muscle action duration $(4 \mathrm{~s}: 2 \mathrm{~s} \times 2 \mathrm{~s}: 4 \mathrm{~s})$ and designed to be performed until muscle failure. It was hypothesized that the protocol with the greater time in the concentric action would show greater EMG amplitude, BLC, MNR, and EMGMF reductions than the protocol with the greater time in the eccentric action.

\section{Methods}

\section{Participants}

The sample size was established a priori following the recommendations of Beck (2013), using data from Lacerda et al. (2017). In this study, it was found that the effect size $=0.39$ (Cohen's $d$ ) for the triceps brachii EMG amplitude at the $5^{\text {th }}$ repetition of the $3^{\text {rd }}$ set (Protocols $4 \mathrm{~s}: 2 \mathrm{~s}$ and $2 \mathrm{~s}: 4 \mathrm{~s}$ ). This value, when inserted into the software $G^{*}$ Power (version 3.1.9.2; Heinrich Heine Universität Düsseldorf, DE, Germany) with power $=0.80$ and $\alpha=0.05$, showed the number of required participants was 16 . Thus, 17 males (1 extra for possible sample loss) with strengthtraining experience (mean \pm standard deviation [SD]: age $=24.6 \pm 2.9 \mathrm{yrs}$; body height $=174.9 \pm 6.8$ $\mathrm{cm}$; body mass $=78 \pm 9.1 \mathrm{~kg}$; resistance training experience $=2.81 \pm 3.40 \mathrm{yrs}$; the number of training sessions per week $=4.4 \pm 1.06 ; 1 \mathrm{RM}$ performance on Smith machine bench press exercise $=90.08 \pm 15.1 \mathrm{~kg}$ ) and no history of injury were enrolled in this study. Before participation in the study, written consent was obtained from each participant after being informed of the procedures, risks, and benefits of the investigation. The design and protocol of the study conformed to the ethical standards established in the Declaration of Helsinki and were approved by the ethics committee of the Federal University of Minas Gerais, Brazil.

\section{Design and Procedures}

This study used a crossover design (counterbalanced) to compare the EMG, BLC, and the MNR performed to failure in two resistance training protocols differentiated by concentric and eccentric contraction times, but with the same repetition duration. It is worth noting that these protocols have already been used in a previous study, but in that study, participants were not taken to muscle failure (Lacerda et al., 2017). Each volunteer participant attended the laboratory on 4 different days (experimental sessions 1 to 4 ), each separated by at least 48 hours. The same data collection schedule was maintained for each participant across all sessions.

Experimental sessions 1 and 2: Following the assessment of body height and mass with a stadiometer (precision of $1 \mathrm{~mm}$ and $100 \mathrm{~g}$, respectively) (Welmy®, São Paulo, Brazil), participants were positioned on a Smith machine bench press (Master ${ }^{\circledR}$, Belo Horizonte, MG, Brazil). The position of the hands and head were self-determined and recorded for the following sessions. The $1 \mathrm{RM}$ test was performed during the first and second sessions aiming to familiarize participants with its procedures and to define the resistance for the following sessions, respectively. Before initiating the 1RM test, all volunteers performed 10 repetitions, lifting only the bar mass $(20 \mathrm{~kg})$ in a slow speed movement. Two minutes later, the test began with an eccentric muscle action by lowering the bar until it touched the sternum (minimum time), followed by a concentric muscle action, which involved the full extension of the elbows, defining the exercise range of motion. The 1RM test was determined within a maximum of 6 attempts, in which the load was gradually increased, with a 5-min rest interval between each attempt (Lacerda et al., $2015,2017)$. When the participant could not perform a complete repetition (full range of motion), the prior load lifted was recorded representing the 1RM test result. Participants were also familiarized with the use of a metronome $\left(60 \mathrm{~b} \cdot \mathrm{min}^{-1}\right)$ by performing the concentric and eccentric phases for each training protocol that would be used during experimental sessions 3 and 4 .

Experimental sessions 3 and 4: The protocols consisted of 3 sets at $60 \%$ of the $1 \mathrm{RM}$, a 3-min rest interval between sets with 6-s repetition duration performed until concentric failure. In the first protocol, the 6-s repetition duration was configured with $2 \mathrm{~s}$ of concentric muscle action and $4 \mathrm{~s}$ of eccentric muscle action 
(Protocol 2 s: 4 s). In the second protocol, the concentric and eccentric muscle actions were performed for $4 \mathrm{~s}$ and $2 \mathrm{~s}$, respectively (Protocol 4 s: $2 \mathrm{~s}$ ). As concentric failure is achieved before full elbow extension, the last successful repetition was considered as the repetition performed just prior to concentric failure.

A calibrated electrogoniometer (Noraxon USA, Scottsdale, AZ USA) was affixed on the left elbow of participants using double-sided adhesive tape and elastic bands. Once stored, the raw electrogoniometer data were converted into angular displacement data and filtered through a $4^{\text {th }}$-order Butterworth low-pass filter with a cut-off frequency of $10 \mathrm{~Hz}$. The electrogoniometer was also used to determine the elbow range of motion and the muscle action duration. The eccentric duration corresponded to the period between the minimum and maximum angular positions, while the concentric duration corresponded to the maximum and minimum angular positions.

The surface electromyography procedures were based on Surface Electromyography for the Non-Invasive Assessment of Muscles (SENIAM) standards for the triceps brachii (Hermes et al., 1992) and on other recommendations for the pectoralis major (Lagally et al., 2004). Bipolar surface electrodes (Ag/AgCl; Red Dot 2223; 3M, Maplewood, MN USA) were placed parallel to the muscle fibres on each participant's right pectoralis major (sternal portion) and triceps brachii (long head portion) muscles. The skin area was shaved and cleaned with alcohol and a cotton pad before placing the electrodes. The electrodes were placed in pairs, $2 \mathrm{~cm}$ apart from their centres at the point of the greatest muscle area. The ground electrode was fixed at the olecranon.

The electromyographic data acquisition was amplified 1000 times and filtered $\left(2^{\text {nd }}-\right.$ order Butterworth band-pass filter of $20-500 \mathrm{~Hz}$ ) to calculate the EMG amplitude by the root mean square (EMGrms). Before starting each experimental session, participants were asked to perform 3 maximum voluntary isometric contractions (MVIC) at $90^{\circ}$ of elbow flexion (controlled by the electrogoniometer); these were used as the reference for the normalization of the subsequent measurements (normalization test) (Lacerda et al., 2017). The bar was fixed by two chains at the extremities, and the highest values found during the 3 MVIC attempts were used as reference for the normalization of the subsequent measurements. The MVIC manoeuvres were performed with 5-s duration each with a 2-min rest interval between trials (Lacerda et al., 2017). Finally, the mean repetition EMGrms obtained during each protocol was calculated, and these values were divided by the respective reference values previously described, generating the normalized EMGRMs per repetition.

The same normalization procedure was adopted for the frequency domain analysis and the EMGMF values obtained from each repetition were normalized by the highest frequency values detected during the three MVIC attempts of each data collection session. The EMGMF was calculated using the discrete-time short-time Fourier transform and applying the Fourier transform in a fixed window size (the Hamming window, $50 \mathrm{~ms}$ ) on the electromyographic signal of each repetition. The frequency resolution was $20 \mathrm{~Hz}$. The EMGMF was selected because it adequately represents the power spectrum of the signal (Kwatny et al., 1970). The EMG and electrogoniometer signals were synchronized and converted using an A/D board (Biovision, Wehrheim, Germany) and sampled at a frequency of $1000 \mathrm{~Hz}$. Appropriate software (DASYLab 11.0; Measurement Computing Corporation, Norton, MA USA) was used to record and treat the data.

Blood samples were collected with the objective of measuring the BLC at different moments: immediately before the training protocol (pre); during the training protocol (1 $\mathrm{min}$ after each set); and after the training protocol $(3,6$, 9 , and $12 \mathrm{~min}$ post-exercise). Obtaining several blood samples takes into account the delay in time for lactate accumulation and lactic acid diffusion into the blood (Scott, 2006). Thus, a greater number of post-exercise measurements helps determine possible differences in BLC (Crewther et al., 2006). Blood samples were collected from a puncture to each participant's left earlobe using sterile disposable lancets. The earlobe was cleaned with neutral soap and water and then sterilized with $70 \%$ alcohol before puncturing. A $30 \mu \mathrm{L}$ sample of blood was collected into heparinized capillary tubes, which were transferred into other tubes containing $60 \mathrm{~mL}$ of $1 \%$ sodium fluoride and then stored, for no longer than 3 months, in a refrigerator maintained at a temperature of $-20^{\circ} \mathrm{C}$. Subsequently, the samples were thawed and 
analysed in duplicate on the YSI Sport 1500 Lactate Analyzer (YSI [now Xylem, Inc.], Rye Brook, NY USA).

\section{Statistical Analysis}

EMGrMs, EMGMF, and BLC data were assessed via an analysis of variance (ANOVA) with repeated measures, and significant differences $(p<0.05)$ were evaluated with a post hoc Tukey's range test using the statistical program Sisvar ${ }^{\circledR}$ (Ferreira, 2011). The normality and homogeneity of variance were verified using Shapiro-Wilk and Mauchly's sphericity tests, respectively. For the analysis of the normalized EMGRMs and EMGMF data, the values from the first, middle, and the last repetitions (beginning, middle and ending) of each set were used. The middle repetition was represented by the median repetition number of the set (i.e. if 8 repetitions were performed by a participant in a set, the normalized EMGrms values of repetitions 4 and 5 were summed and divided by 2); the EMG analysis of the repetition was done according to a previous study (Jenkins et al., 2015). A 3-way (protocol $\times$ set $\times$ repetition) ANOVA with repeated measures was used to assess the normalized EMGrms and EMGMF data. The intraclass correlation coefficients (ICC $[3, \mathrm{k}]$ ) found in the normalization test of experimental sessions 3 and 4 for EMGrms were 0.90 and 0.94 for the pectoralis major and the triceps brachii, respectively. The EMGMF was 0.93 for both the pectoralis major and the triceps brachii. Eta squared $\left(n^{2}\right)$ values were reported to reflect the magnitude of the differences in each treatment $($ small $=0.01$, medium $=0.06$, and large $=0.14)$ (Cohen, 1988). For the comparison of the BLC response, a 2-way (protocol $\times$ time) ANOVA with repeated measures was used to assess lactate concentrations across time. To compare the MNR between protocols and sets, the ANOVA-type rank test with the post hoc Dunn's Multiple Comparison Test was used. Both procedures were performed using $\mathrm{R}$ software (The $\mathrm{R}$ Foundation, www.r-project.org).

\section{Results}

Mean concentric and eccentric duration for Protocol $4 \mathrm{~s}: 2 \mathrm{~s}$ was $3.98 \pm 0.12 \mathrm{~s}$ and $2.07 \pm$ $0.13 \mathrm{~s}$, respectively. For Protocol 2 s: 4 s, the duration times were $2.16 \pm 0.18 \mathrm{~s}$ and $3.82 \pm 0.17 \mathrm{~s}$ for concentric and eccentric phases, respectively.
Regarding the MNR, ANOVA-type rank testing found only a main effect for set $\left(\mathrm{H}_{1,2}=70.16, p=\right.$ $0.001, \eta^{2}=0.22$ ). The Dunn's test showed that more repetitions were performed in the first set compared to the second and third sets, as detailed in Table 1. There were no significant interactions. EMGRMS

For the pectoralis major muscle, there was a significant main effect for the protocol $\left(F_{1,96}=\right.$ 4.682, $\left.p=0.03, \mathrm{\eta}^{2}=0.03\right)$, set $\left(F_{2,96}=3.22, p=0.04, \mathrm{\eta}^{2}\right.$ $=0.04)$, and repetition $\left(F_{2,192}=3.22, p=0.04, \eta^{2}=\right.$ 0.15). Post hoc analyses indicated that Protocol $4 \mathrm{~s}$ : $2 \mathrm{~s}$ had a significantly higher muscle activation than Protocol 2 s: 4 s. Also, the mean normalized EMGrms of the second and third sets were similar, but values of the third set were higher than of the first. Finally, the mean normalized EMGrus of the last repetition was higher than the first and middle repetitions. Figure $1 \mathrm{~A}$ shows the normalized EMGrus response for the pectoralis major. There were no significant interactions between factors.

For the triceps brachii and the mean normalized EMGRMs there was a significant main effect only for repetition $\left(F_{2,192}=247.35, p<0.01\right.$, $\mathrm{\eta}^{2}$ $=0.15$ ). A Tukey's post hoc analysis found that the last repetition had a significantly higher muscle activation than the first and middle repetitions. Moreover, the middle repetition showed higher muscle activation than the first repetition. Figure $1 \mathrm{~B}$ shows the normalized EMGrus values for the triceps brachii. There were no significant interactions between factors.

EMGMF

Concerning the normalized EMGMF data for the pectoralis major, ANOVA indicated a significant main effect only for repetition $\left(F_{2,192}=\right.$ 247.31, $\left.p<0.001, \mathrm{n}^{2}=0.15\right)$. Tukey's post hoc testing showed a significantly lower normalized EMGMF in the last repetition compared to the first and middle repetitions and the middle repetition presented a significantly lower normalized EMGMF value than the first repetition. Figure 2A presents the normalized EMGMF data for the pectoralis major. There were no significant interactions between factors.

Analysis of the normalized EMGMF values for the triceps brachii resulted in a significant interaction for the protocol and repetition $\left(F_{2,92}=\right.$ 3.248; $p=0.04 ; \eta^{2}=0.06$ ). Post hoc analysis indicated that Protocol 4 s: 2 s had significantly 
lower normalized EMGMF values in the middle and last repetitions compared to Protocol 2 s: $4 \mathrm{~s}$. Moreover, a significant interaction was found for the set and repetition $\left(F_{4,192}=3.941 ; p<0.001 ; \eta^{2}<\right.$ 0.001 ) with the last repetition presenting the lowest normalized EMGMF value followed by the middle repetition during the first two sets. However, at the third set, no significant difference in the normalized EMGMF values between the first and middle repetitions were detected, and the values in these two repetitions were significantly higher than that in the last repetition.
Additionally, there were significant main effects for the protocol and repetition $\left(F_{1,96}=4.85, p=0.03\right.$, $\eta^{2}=0.03$ and $F_{2,192}=52.32, p<0.001, \eta^{2}=0.04$, respectively). Figure $2 \mathrm{~B}$ shows the normalized EMGMF for the triceps brachii.

Blood lactate concentration

There was a main effect only for time $\left(F_{7,224}\right.$ $=225.229, p<0.001, \eta^{2}=0.09$ ) with all BLC post exercise responses being significantly higher when compared to resting values. The BLC response is presented in Table 2.

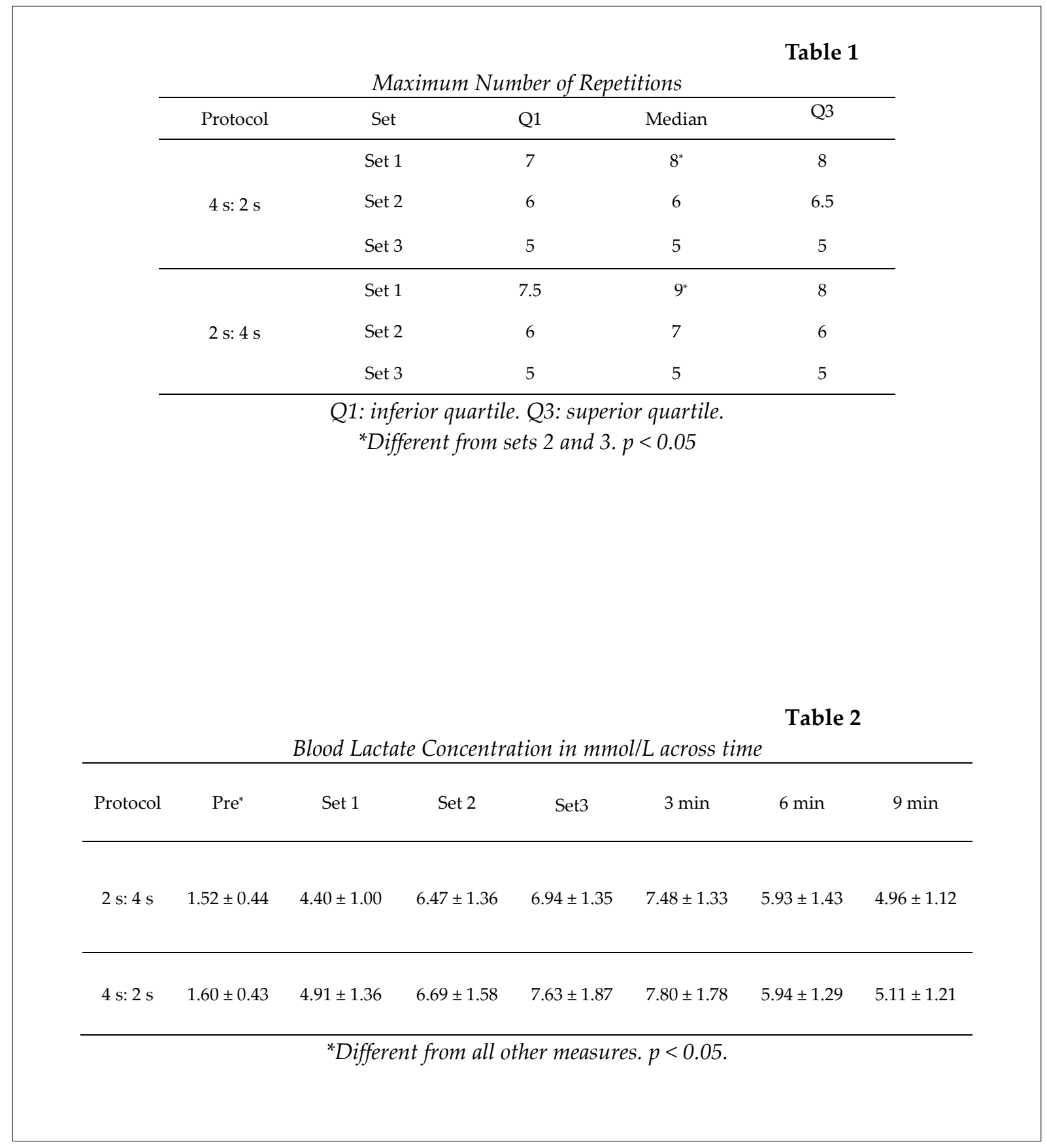




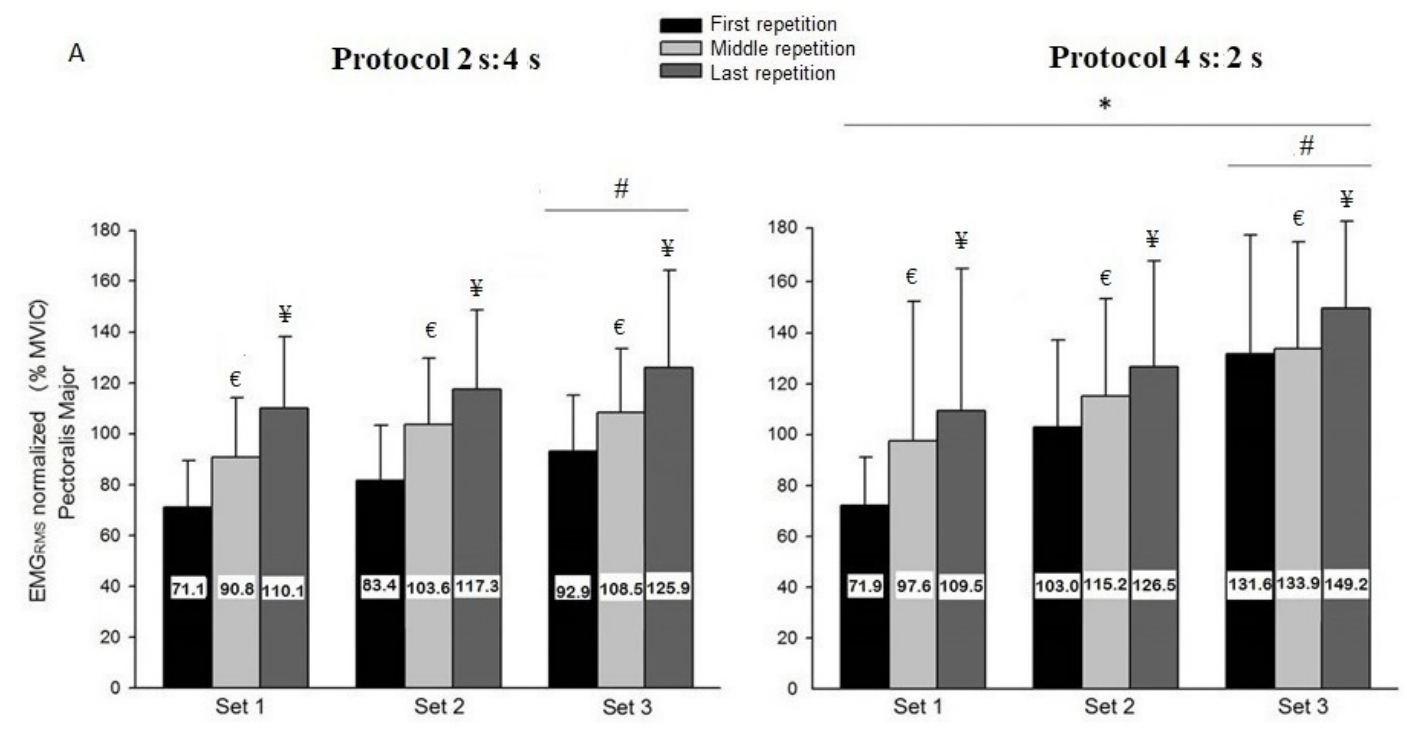

B Protocol 2 s:4s

Protocol $4 \mathrm{~s}: 2 \mathrm{~s}$
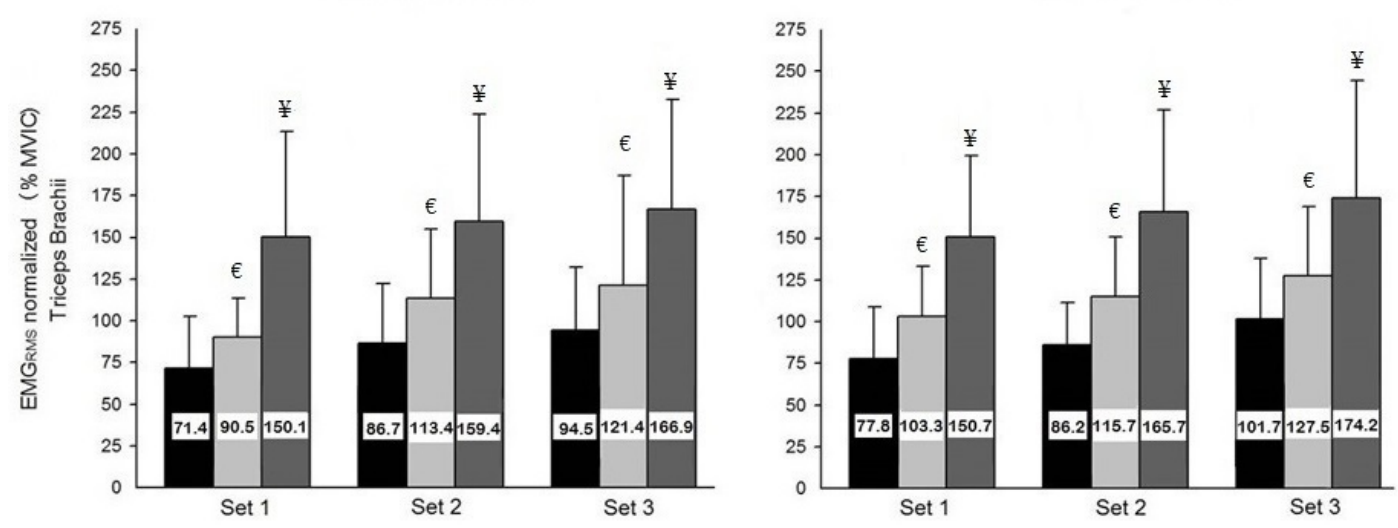

Figure 1

Pectoralis major (A) and triceps brachii (B) muscle activation.

EMGRMS: root mean square of the electromyographic signal. MVIC: Maximum voluntary isometric contraction. Standard errors: vertical lines; * different from Protocol $2 \mathrm{~s}: 4 \mathrm{~s} ;$; different from Set $1 ;{ }^{*}$ different from the first and middle repetition; $\epsilon^{\epsilon}$ different from the first repetition. $p<0.05$ 
A Protocol $2 \mathrm{~s}: 4 \mathrm{~s}$ Mirst repetition

Protocol $4 \mathrm{~s}: 2 \mathrm{~s}$
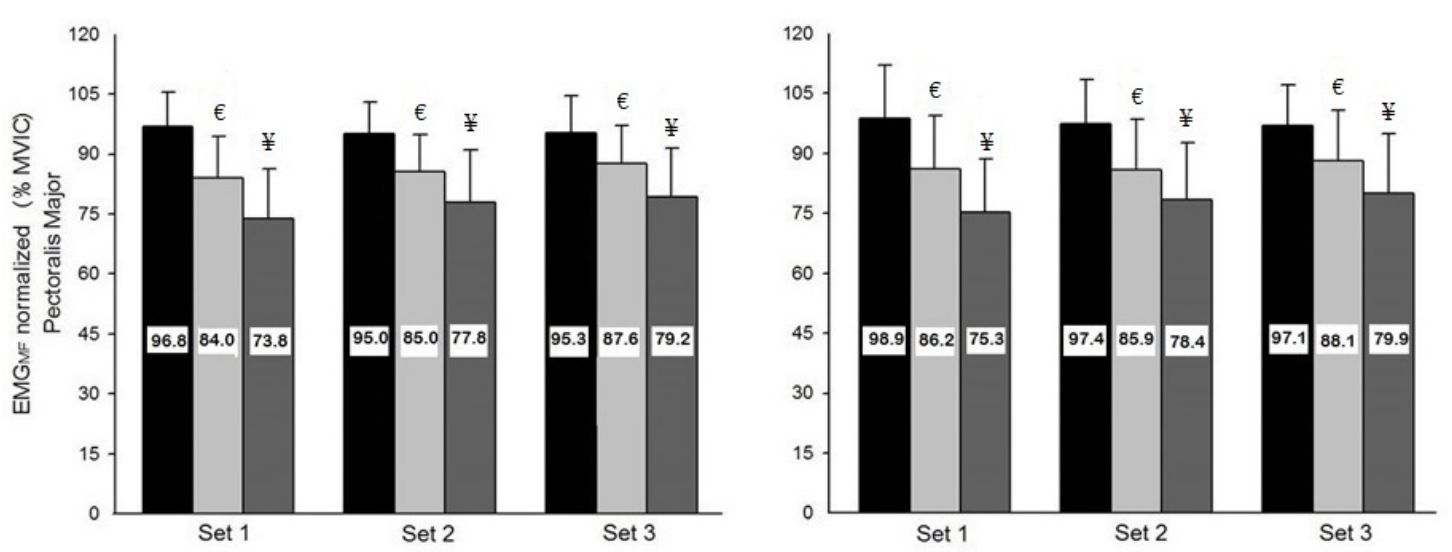

Protocol $2 \mathrm{~s}: 4 \mathrm{~s}$

Protocol $4 \mathrm{~s}: 2 \mathrm{~s}$

$\psi$

B
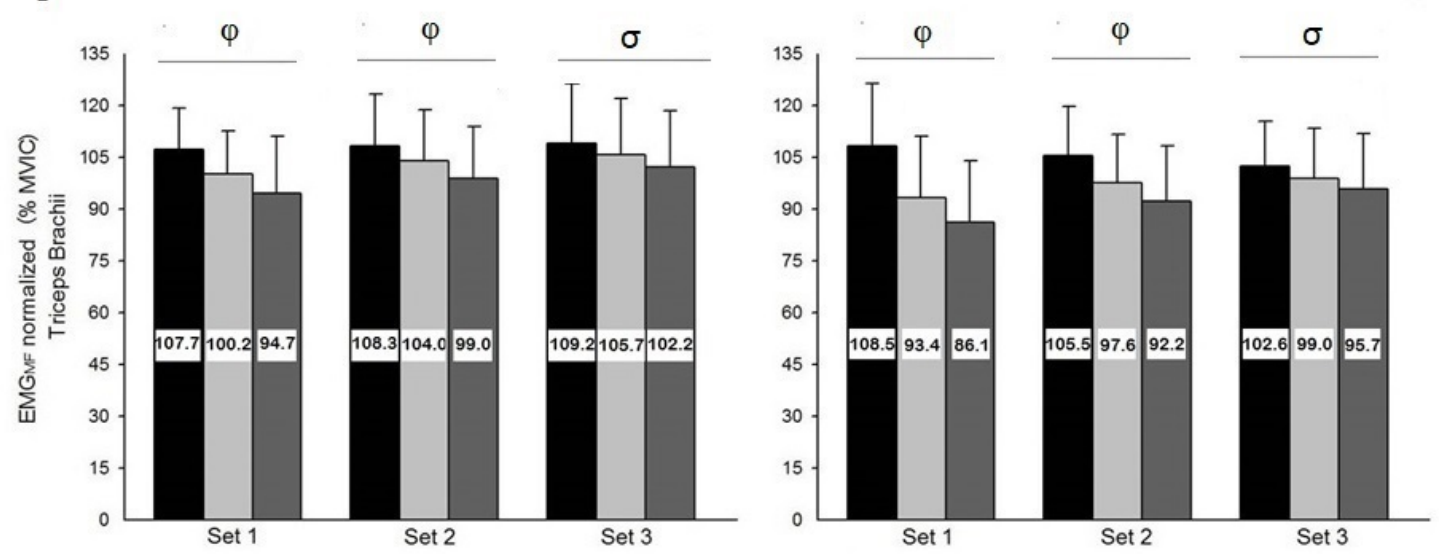

Figure 2

Electromyographic frequency of the pectoralis major $(A)$ and triceps brachii $(B)$ muscles.

EMGMF: mean electromyographic frequency signal. MVIC: Maximum voluntary isometric contraction. Standard errors: vertical lines; $\psi$ different from Protocol 2 s: 4 s at the middle and last repetitions; $\varphi$ different among repetitions at the respective set; $\sigma$ last repetition different from the first and middle repetition at the respective set; ${ }^{¥}$ different from the first and middle repetition; $\epsilon$ different from the first repetition. $p<0.05$. 


\section{Discussion}

The normalized EMGRMS response for the pectoralis major was greater for Protocol 4 s: 2 $\mathrm{s}$, suggesting that there was a greater increase in muscle activation when more time was spent in the concentric action (Duchateau and Enoka, 2008; Lacerda et al., 2017). It is likely that Protocol 4 s: 2 $\mathrm{s}$ imposed a greater demand on the central nervous system since there was a need for a greater activation of higher threshold motor units (Allen et al., 2008; Zajac et al., 2015) or an increase in motor unit synchronization (Dartnell et al., 2008). However, caution should be considered with this interpretation as the effect size for this response was quite low ( $\mathrm{\eta} 2=0.03$ ). Additionally, the last two sets and the last repetition performed presented higher normalized EMGRMS responses compared to the first set and the previous repetitions, respectively. These findings corroborate with previous research that identified an increase in muscle activation across sets and repetitions when an exercise was performed until muscle failure (Jenkins et al., 2015). Increased levels of muscle fatigue demand an increase in higher threshold motor unit recruitment in an attempt to maintain force production (Conwit et al., 2000). The present results add evidence to this line of reasoning since the elevation of neuromuscular demand across sets was accompanied by continuous increases in BLC across sets and a reduction in the EMGMF response across the repetitions.

However, for the triceps brachii muscle, Protocol 4 s: 2 s did not present a higher EMGRMS response than Protocol 2 s: 4 s. This result indicates that when the Smith bench press exercise was completed to muscle failure, the $2 \mathrm{~s}$ difference between protocols did not influence the normalized triceps brachii EMGRMS response. Previous research showed greater triceps brachii muscle activation for Protocol $4 \mathrm{~s}$ : $2 \mathrm{~s}$ at the fifth repetition in comparison to Protocol $2 \mathrm{~s}$ : $4 \mathrm{~s}$ (Lacerda et al., 2017), however, the protocols were not completed to muscle failure. In addition, the EMGRMS response of the pectoralis major and the triceps brachii muscles shows evidence that the considered prime movers may present different neurophysiological responses when the Smith bench press exercise is performed to muscle failure (Dugdale et al., 2017).

These non-uniform behaviours between the prime movers could be explained by several factors. Firstly, as the bench press is a multi-joint exercise, at a given range of motion, the different prime movers may be at different percentiles of their optimal muscle length to produce force. Consequently, those muscles may contribute with different levels of force output along the range of motion that might result in different levels of muscle force production and activation (Tillar and Saeterbakken, 2013). Another factor may be associated with the different anatomical functions performed by biarticular muscles, such as the triceps brachii, that result in a greater degree of variability in the relationship between EMG amplitude and force response (Luera et al., 2014). It is possible that these factors may also have influenced the non-uniform behaviour of EMGMF between the pectoralis major and the triceps brachii muscles.

To explain the similar EMG amplitude response from the triceps brachii muscle between protocols, a previous study showed that throughout the range of motion of the bench press exercise, the elevation of the triceps brachii activation level occurred only after the sticking point (Tillar and Saeterbakken, 2013), the point of greatest mechanical disadvantage (Kompf and Arandjelovic, 2016). In this sense, Protocol 4 s: 2 s required a greater time under tension during the concentric action than Protocol 2 s: 4 s, in a way that seems to be propitious to elevate the EMG amplitude (Duchateau and Enoka, 2008; Lacerda et al., 2017). However, Protocol 2 s: 4 s demanded a greater production of force due to the higher velocity required for the concentric action, which may also elevate the EMG amplitude (Sampson et al., 2014). Thus, perhaps the greater concentric duration of Protocol $4 \mathrm{~s}: 2 \mathrm{~s}$ and the greater force of Protocol 2 s: 4 s were stimuli that resulted in a similar normalized EMGRMS response for the triceps brachii muscle.

Results from the normalized EMGMF response for the pectoralis major muscle indicated no significant interactions or significant main effects for protocols or sets, only across repetitions. A decrease in the EMGMF frequency has been associated with a decreased velocity of the nerve impulse conduction and muscle cell membrane excitability (Conwit et al., 2000) due to imbalances of intra and extracellular ionic concentrations, inactivation of $\mathrm{Ca} 2+$ channels, and 
increased local acidosis (Brody et al., 1991). In the present study, the differences in the configuration of muscle action duration between protocols were not sufficient to cause a greater decrease in EMGMF for the pectoralis major in any of the protocols investigated. Thus, despite Protocol $4 \mathrm{~s}$ : 2 s evoking a higher normalized EMGRMS response than Protocol 2 s: 4 s, the neuromuscular fatigue, demonstrated by the EMGMF response, was similar between protocols. This result shows that a greater increase of muscle activation may not be followed by a greater reduction in the EMGMF, which corroborates a previous study (Jenkins et al., 2015). The metabolic accumulation may, at least in part, impair EMGMF by reducing the membrane excitability (Brody et al., 1991). The analogue metabolic accumulation seen in the protocols, regarding the BLC response, may be associated to the similar decrease in the EMGMF response. Additionally, it appears that the 3-min rest intervals between the sets were sufficient for homeostasis to be re-established for this muscle (Allen et al., 2008).

Accompanying the significant reductions in EMGMF response across repetitions within sets for both protocols and muscles, there were increases in BLCs and neuromuscular activation. These responses indicate a large physiological demand that might have caused alterations in cell excitability (Allen et al., 2008) leading to a decreased EMGMF response across the repetitions. In addition, both protocols caused significant increases in BLCs in all measurements compared to resting values, however, no significant differences in the BLC response were detected between protocols. Goto et al. (2009) reported that the protocol that resulted in the greatest number of repetitions also produced the highest BLC response. In the present study, both protocols resulted in similar numbers of repetitions and similar BLC response across sets. The explanation for the dissimilar findings between these two studies may be due to the muscle action duration. The smaller difference between the muscle action duration (2s) of the present study in comparison to the 4-s difference used in the study by Goto et al. (2009) may have been insufficient to elicit a different BLC response between the two protocols. Perhaps the two protocols were based on similar anaerobic energy demands (Scott, 2006).

Relative to the MNR completed in both protocols, participants completed significantly more repetitions in the first set compared to the second and third sets, indicating an accumulation of fatigue that affected the subsequent sets. However, there was no significant difference between the MNR between protocols, contrary to the initial hypothesis. Despite the differences in the EMGRMS response for the pectoralis major and the EMGMF response for the triceps brachii for the two protocols, the BLC response and the MNR completed were the same. These findings, taken together, may indicate that the central nervous system may have developed different strategies for muscle activation in order to maintain force production and to overcome fatigue that was generated by the protocols, resulting in an increased ability to maximize the number of successful repetitions before muscle failure.

\section{Conclusions}

In summary, this study demonstrated that protocols with different muscle action duration, but similar repetition duration may differentially influence the muscle activation levels and the neuromuscular fatigue patterns in two prime mover muscles involved in the execution of the Smith machine bench press exercise without affecting the BLC response and the MNR performed. Moreover, this study also provides evidence that a small 2-s difference between muscle action duration used in the two protocols of this study, designed to perform repetitions to muscle failure, was not sufficient to alter the training volume between the two protocols.

\section{Acknowledgements}

We thank the Coordenação de Aperfeiçoamento de Pessoal de Nível Superior - Brasil (CAPES), finance code 001, Conselho Nacional de Desenvolvimento Científico e Técnológico (CNPq), and Fundação de Amparo à Pesquisa do Estado de Minas Gerais (FAPEMIG) for financial support. We also thank the PróReitoria de Pesquisa (PRPq) da Universidade Federal de Minas Gerais for research support. 


\section{References}

Allen DG, Lamb GD, Westerblad H. Skeletal Muscle Fatigue: Cellular mechanisms. Physiol Rev, 2008; 88: 287332

Batalha N, Dias S, Marinho D, Parraca J. The Effectiveness of Land and Water Based Resistance Training on Shoulder Rotator Cuff Strength and Balance of Youth Swimmers. J Hum Kinet, 2018; 62: 91-102

Beck TW. The importance of a priori sample size estimation in strength and conditioning research. J Strength Cond Res, 2013; 27: 2323-2337

Brody LR, Pollock MT, Roy SH, De Luca CJ, Celli B. PH-induced effects on median frequency and conduction velocity of the myoelectric signal. J Appl Physiol, 1991; 71: 1878-1885

Cohen J. Statistical Power Analysis for the Behavioral Sciences. Hillsdale, NJ: Lawrence Erlbaum Associates; 1988

Conwit RA, Stashuk D, Suzuki H, Lynch N, Schrager M, Metter EJ. Fatigue effect on motor unit activity during submaximal contractions. Arch Phys Med Rehabil, 2000; 81: 1211-1216

Crewther B, Cronin J, Keogh K. Possible stimuli for strength and power adaptation: acute metabolic responses. Sports Med, 2006; 36: 65-78

Dartnell T, Nordstrom MA, Semmler JG. Motor unit synchronization is increased in biceps brachii after exercise-induced damage to elbow flexor muscles. J Neurophysiol, 2008; 99: 008-1019

Duchateau J, Enoka RM. Neural control of shortening and lengthening contractions: influence of task constraints. J Neurophysiol, 2008; 586: 5853-5864

Dugdale AH, Hunter A, Di Virgilio T, Macgregor LJ, Hamilton L. Influence of the "Slingshot" bench press training aid on bench press kinematics and neuromuscular activity in competitive powerlifters. $J$ Strength Cond Res, 2019; 33: 327-336

Fang Y, Siemionow V, Sahgal V, Xiong F, Yue GH. Greater movement-related cortical potential during human eccentric versus concentric muscle contractions. J Neurophysiol, 2001; 86: 1764-1772

Ferreira DC. Sisvar: a computer statistical analysis system. Ciênc Agrotec, 2011; 35: 1039-1042

Goto K, Ishii N, Kizuka T, Kraemer RR, Honda Y, Takamatsu K. Hormonal and metabolic responses to slow movement resistance exercise with different durations of concentric and eccentric actions. Eur J Appl Physiol, 2009; 106: 731-739

Hermes HJ, Brugen TA, Baten CT, Rutten WL, Boom HB. The median frequency of the surface EMG power spectrum in relation to motor unit firing and action potential properties. J Electromyogr Kinesiol, 1992; 2: $15-25$

Hunter SK, Duchateau J, Enoka RM. Muscle fatigue and the mechanisms of task failure. Exerc Sport Sci Rev, 2004; 32: 44-49

Jenkins ND, Housh TJ, Bergstrom HC, Cochrane KC, Hill EC, Smith CM, Johnson GO, Schmidt RJ, Cramer JT. Muscle activation during three sets to failure at 80 vs. 30\% 1RM resistance exercise. Eur J Appl Physiol, 2015; 115: 2335-2347

Kompf J, Arandjelovic O. Understanding and overcoming the sticking point in resistance exercise. Sports Med, 2016; 46: 751-762

Kwatny E, Thomas DH, Kwatny HG. An application of signal processing techniques to the study of myoelectric signals. IEEE Trans Biomed Eng, 1970; 17: 303-313

Lacerda LT, Costa SG, Lima FV, Martins-Costa HC, Diniz RC, Andrade AGP, Peixoto GHC, Bemben MG, Chagas $\mathrm{MH}$. Longer concentric action increases muscle activation and neuromuscular fatigue responses in protocols equalized by repetition duration. J Strength Cond Res, 2019; 33: 1629-1639

Lacerda, LT, Martins-Costa, HC, Diniz, RCR, Lima, FV, Andrade, AGP, Tourino, F D, Bemben, MG, Chagas, $\mathrm{MH}$. Variations in repetition duration and repetition numbers influence muscular activation and blood lactate response in protocols equalized by time under tension. J Strength Cond Res, 2016; 30: 251258

Lagally KM, McCaw ST, Young GT, Medema HC, Thomas DQ. Ratings of perceived exertion and muscle activity during the bench press exercise in recreational and novice lifters. J Strength Cond Res, 2004; 18: 359-364 
Lago-Fuentes C, Rey E, Padrón-Cabo A, Rellán-Guerra AS, Fragueiro-Rodríguez A, García-Núñez J. Effects of Core Strength Training Using Stable and Unstable Surfaces on Physical Fitness and Functional Performance in Professional Female Futsal Players. J Hum Kinet, 2018; 65: 213-224

Lehnert M, Sigmund M, Lipinska P, Vařeková R, Hroch M, Xaverová Z, Stastny P, Háp P, Zmijewski P. Training-induced changes in physical performance can be achieved without body mass reduction after eight week of strength and injury prevention oriented programme in volleyball female players. Biol Sport, 2017; 34: 205-213

Luera MJ, Stocs MS, Chappell AD. Electromyographic amplitude vs. concentric and eccentric squat force relationships for monoarticular and biarticular thigh muscles. J Strength Cond Res, 2014; 28: 328-338

Maszczyk A, Wilk M, Krzysztofik M, Gepfert M, Zając A, Petr M, Stastny P. The influence of the level of experience in muscle strength training on the value of time under tension. Biol Sport, 2020; 37(1): 79-83

Ratamess NA, Alvar BA, Evetoch TK, Housh TJ, Kibler WB, M.D, Kraemer WJ, Triplett TT. American College of Sports Medicine position stand. Progression models in resistance training for healthy adults. Med Sci Sports Exerc, 2009; 41: 687-708

Sampson JA, Donohoe A, Groeller H. Effect of concentric and eccentric velocity during heavy-load nonballistic elbow flexion resistance exercise. J Sci Med Sport, 2014; 17(3): 306-311

Scott CB. Contribution of blood lactate to the energy expenditure of weight training. J Strength Cond Res, 2006; 20: 404-411

Tillar RV, Saeterbakken AH. Fatigue effects upon sticking region and electromyography in a six-repetition maximum bench press. J Sports Sci, 2013; 31: 1823-1830

Usui S, Maeo S, Tayashiki K, Natakatani M, Kanehisa H. Low-load slow movement squat training increases muscle size and strength but not power. Int J Sports Med, 2016; 37: 305-312

Wakahara T, Fukutani A, Kawakami Y, Yanai, T. Nonuniform muscle hypertrophy: its relation to muscle activation in training session. Med Sci Sports and Exerc, 2013; 45: 2158-2165

Watanabe Y. Madarame H, Ogasawara R, Nakazato K, Ishii N. Effect of very low-intensity resistance training with slow movement on muscle size and strength in healthy older adults. Clin Physiol Funct Imaging, 2014; 34: 463-470.

Zając A, Chalimoniuk M, Maszczyk A, Gołaś A, Lngfort J. Central and Peripheral Fatigue During Resistance Exercise - A Critical Review. J Hum Kinet, 2015; 49: 159-69

\title{
Corresponding author:
}

\author{
Mauro H. Chagas, PhD \\ Weight Training Laboratory \\ Universidade Federal de Minas Gerais \\ Av. Antônio Carlos, 6627, \\ Belo Horizonte, Brazil. \\ Phone: (+55)31 31270-901, \\ E-mail: mauroufmg@hotmail.com
}

